\title{
Increased vascular permeability after cardiopulmonary bypass in patients with diabetes is associated with increased expression of vascular endothelial growth factor and hepatocyte growth factor
}

\author{
Sirisha Emani, PhD, Basel Ramlawi, MD, Neel R. Sodha, MD, Jian Li, MD, PhD, \\ Cesario Bianchi, MD, PhD, and Frank W. Sellke, MD
}

\begin{abstract}
Background: Several inflammatory mediators such as vascular endothelial growth factor and hepatocyte growth factor are known to play a critical role in the regulation of vascular permeability and angiogenesis. We studied the serum levels of growth factors and gene expression profiles of genes involved in growth factor signaling in the peripheral blood of patients with and patients without diabetes following cardiopulmonary bypass and cardioplegic arrest.
\end{abstract}

\begin{abstract}
Methods: Serum and total RNA were obtained from the blood samples collected from patients with diabetes and matched patients without diabetes $(n=7$ patients each) who had coronary artery bypass graft before and 6 hours and 4 days after cardiopulmonary bypass/cardioplegic arrest. The cytokine panel, consisting of growth factors such as vascular endothelial growth factor, hepatocyte growth factor, fibroblast growth factor, and epidermal growth factor, was quantified in patients with diabetes and patients without diabetes before and 6 hours and 4 days post-cardiopulmonary bypass/cardioplegic arrest using multiplex cytokine quantification system. cDNA microarray analysis was performed and fold-change was calculated.
\end{abstract}

Results: Length of hospitalization (10 vs 6 days; $P=.04$ ) and weight gain (5 vs $2.5 \mathrm{~kg} ; P=.001)$ were significantly greater for patients with diabetes compared with patients without diabetes. The serum levels of vascular endothelial growth factor and hepatocyte growth factor were significantly elevated in patients with diabetes when compared with patients without diabetes before versus 6 hours post-cardiopulmonary bypass/cardioplegic arrest. In addition, significantly elevated mRNA expression of hypoxia-inducible factor- $1 \alpha$, cyclic adenosine monophosphate response element binding protein, and E1A binding protein p300 (more than twofold) was observed 4 days post-cardiopulmonary bypass/cardioplegic arrest exclusively in patients with diabetes.

Conclusions: The differential profile of gene and protein expression of growth factors and their related genes in patients with diabetes and patients without diabetes could be associated with increased edema and weight gain in patients with diabetes after cardiopulmonary bypass/cardioplegic arrest.

Cardiovascular disease is the leading cause of death among individuals with diabetes mellitus (DM), and $\sim 34 \%$ of the patients having coronary artery bypass grafting (CABG) have been reported to have diabetes. ${ }^{1}$ Several adverse surgical outcomes, such as higher perioperative mortality, sternal wound infections, postoperative stroke, and longer hospitalization post-cardiac surgery, have been reported in patients with DM compared with patients without DM.,3

Cardiopulmonary bypass (CPB) during cardiac surgery is associated with extensive inflammatory triggers including

\footnotetext{
From Cardiovascular Surgery, Beth Israel Deaconess Medical Center, Harvard Medical School, Boston, Mass.

This project was funded by NIH HL46716 grant and Irving Bard Memorial Fellowship. S.E. was supported by NIH grant T32 HL07917-07 to Dr Bruce Furie.

Received for publication March 3, 2008; revisions received Dec 8, 2008; accepted for publication Dec 25, 2008.

Address for reprints: Frank W. Sellke, MD, 110 Francis St, Suite 2A, Boston, MA 02215 (E-mail: fsellke@bidmc.harvard.edu).

J Thorac Cardiovasc Surg 2009;138:185-91

0022-5223/\$36.00

Copyright $(c) 2009$ by The American Association for Thoracic Surgery

doi:10.1016/j.jtcvs.2008.12.024
}

the blood-bypass circuit interface, ischemia-reperfusion injury of heart and lungs, and operative trauma. These inflammatory signals induce the activation of microcirculation throughout the body, thus leading to multiorgan dysfunction in the immediate postoperative period. ${ }^{4}$ The other major contributory factor for organ dysfunction after CPB is postoperative hypoxia.

Hypoxic conditions can induce a variety of genes encoding proteins including growth factors, such as vascular endothelial growth factor (VEGF), via hypoxia-inducible factor- $1 \alpha$ (HIF-1 $\alpha$ ), which regulates vascular permeability and cell proliferation. ${ }^{5}$ Growth factors such as insulin-like growth factor, ${ }^{6}$ hepatocyte growth factor (HGF), ${ }^{7}$ keratocyte growth factor, ${ }^{8}$ transforming growth factor- $\beta 1,{ }^{9}$ and also the inflammatory mediators including interleukin-1 and interleukin- $6^{10}$ are known to regulate VEGF synthesis. VEGF is a multifunctional cytokine, overexpressed in hypoxia, and is known to be involved in wound healing, ${ }^{11}$ formation of collateral vessels in ischemic tissue, ${ }^{12}$ chronic inflammatory disorders (psoriasis, rheumatoid arthritis), ${ }^{13}$ diabetic retinopathy, ${ }^{14}$ and tumor growth. ${ }^{15}$ The biologic properties of VEGF include 


\author{
Abbreviations and Acronyms \\ $\mathrm{CABG}=$ coronary artery bypass grafting \\ $\mathrm{CPB}=$ cardiopulmonary bypass \\ CREB = cyclic adenosine monophosphate \\ response element binding protein \\ $\mathrm{CRP}=\mathrm{C}$-reactive protein \\ $\mathrm{DM}=$ diabetes mellitus \\ $\mathrm{EP} 300=\mathrm{E} 1 \mathrm{~A}$ binding protein $\mathrm{p} 300$ \\ HGF = hepatocyte growth factor \\ HIF- $1 \alpha=$ hypoxia-inducible factor- $1 \alpha$ \\ $\mathrm{PCR}=$ polymerase chain reaction \\ RMA $=$ Robust Multichip average \\ VEGF $=$ vascular endothelial growth factor
}

endothelial cell proliferation, ${ }^{16}$ chemotaxis of macrophages and vascular smooth muscle cells, ${ }^{17}$ induction of adhesion molecules (vascular cell adhesion molecule and intercellular adhesion molecule), ${ }^{18}$ and metalloproteinase synthesis. ${ }^{19}$ On the other hand, C-reactive protein (CRP), a potential marker for acute inflammation and a potent independent predictor for cardiovascular diseases including arteriosclerosis, ${ }^{20}$ has been reported to down-regulate VEGF receptors on endothelial cells ${ }^{21}$ and thus may lead to decreased angiogenesis in patients with DM. Acute systemic hypoxia can induce leukocyte extravasation into tissues via VEGF signaling followed by fluid accumulation and thus induces overall weight gain in patients after cardiac surgery.

The mechanisms contributing to the differences in the postsurgical complications observed between patients with DM and patients without DM are not well established. In this study, we evaluated serum levels of several growth factors including VEGF and HGF as well as gene expression profiles of genes involved in growth factor signaling in the peripheral blood of patients with DM and patients without DM following $\mathrm{CPB} /$ cardioplegic arrest. The observed differential quantitative and qualitative protein and gene expressions between patients with DM and patients without DM could provide insight into the observed differences in postoperative inflammatory state between the 2 patient populations.

\section{MATERIALS AND METHODS \\ Subjects}

This prospective cohort study was initiated after being approved by the Beth Israel Deaconess Medical Center Institutional Review Board/Committee on Clinical Investigations. Before enrollment, informed written consent was obtained from all patients interested in participating in the study. The 14 enrolled patients were scheduled for either elective or urgent primary CABG or valvular surgery (aortic, mitral, or both). Because all patients with DM considered for the study had elevated preoperative blood sugar levels $\geq 240 \mathrm{mg} / \mathrm{dL}$, they had preoperative treatment using either an insulin infusion or subcutaneous treatment. Normoglycemic patients without DM received no further glucose treatment presurgery.

\section{Anesthetic And Surgical Technique}

Under general anesthesia, midline sternotomy was performed, and systemic heparin $(100 \mathrm{U} / \mathrm{kg})$ was administered to attain an activated clotting time $>400$. The right atrium and ascending aorta were cannulated, and $\mathrm{CPB}$ was initiated. $\mathrm{CPB}$ circuit consisted of a standard roller pump, a membrane oxygenator, and a $40-\mu \mathrm{m}$ arterial filter. The circuit was primed with crystalloid solution. CPB strategy included the use of mild hypothermia $\left(30^{\circ} \mathrm{C}-34^{\circ} \mathrm{C}\right)$, maintenance of serum glucose levels $\leq 130 \mathrm{mg} / \mathrm{dL}$ with intravenous insulin injections, and $\alpha$-stat $\mathrm{pH}$ monitoring. During CPB, insulin infusion was utilized to maintain normoglycemia, and this strategy was continued in the intensive care unit for 24 hours. Subsequently, subcutaneous insulin was utilized for postoperative glucose control. Pump blood flow was maintained between 2 and $2.4 \mathrm{~L} \cdot \mathrm{min}^{-1} \cdot \mathrm{m}^{-2}$ of body surface area, and mean arterial pressure was maintained between 50 and $90 \mathrm{~mm}$ $\mathrm{Hg}$ with the use of conventional vasoactive medications. Cell-Saver (Haemonetics Corp., Braintree, Mass) and cardiotomy suction were utilized. The decision to administer antifibrinolytic agents (either aprotinin or Amicar) was left to the discretion of the operating team. Cardiac arrest was induced and maintained with antegrade administration of cold-blood hyperkalemic $(25 \mathrm{mmol} / \mathrm{L})$ cardioplegic solution.

\section{Blood Processing and RNA Extraction}

Blood samples were collected in the sterile PAX tubes (Qiagen Inc, Valencia, Calif) from the central venous line after induction of anesthesia but before skin incision preoperatively and 6 hours and 4 days post-CPB/ cardioplegic arrest. Blood samples were centrifuged immediately at $15,000 \mathrm{~g}$ for 15 minutes, and serum/plasma samples were frozen at $-80^{\circ} \mathrm{C}$ until the time of the assay. RNA extraction was performed using PAX gene kit (Qiagen Inc). For microarray analysis, after the quantitative and qualitative assessment of extracted total RNA, single-stranded followed by double-stranded cDNA synthesis was performed. Biotin-labeled cRNA was obtained by in vitro transcription of double-stranded cDNA using AFFI kit (Affymetrix, Santa Clara, Calif). cRNA was further purified, fragmented, hybridized overnight onto Affymetrix gene chips, washed in streptavidin followed by array scanning as previously described. ${ }^{22}$

\section{Microarray Analysis}

Transcriptional profiling was performed on HG-U133 plus 2.0 Affymetrix chips containing $>47,000$ transcripts. The quality of the chips was assessed, and the outliers, calculated using the dChip algorithm, were eliminated from the analysis. Matrix of gene expression values was generated using Robust Multichip average (RMA) normalization statistics (John's Hopkins University, Baltimore, Md). The presence calls of transcripts obtained from Microarray system 5 in conjunction with gene expression values from RMA were used to generate a fusion model to compare the differences in gene regulation postsurgery (6 hours or 4 days) compared with pre-cardiac surgery in patients with DM versus patients without DM ( $n=7$ each group). Changes of more than twofold along with enhanced expression of that transcript in $50 \%$ or more of patients post-cardiac surgery for up-regulated genes or presurgery for down-regulated genes were the major criteria to determine significant differential gene regulation between the subsets $(P<.001)$.

\section{Real-Time Polymerase Chain Reaction}

For real-time polymerase chain reaction (PCR) analysis, one-step reverse transcriptase-PCR reactions were performed using SYBR Green detection in the final volume of $25 \mu \mathrm{L}$. The specific primers for the genes of interest were designed using OligoPerfect software (Invitrogen Inc, Carlsbad, Calif). SYBR Green reactions were performed with SYBR Green PCR Master Mix (Applied Biosystems, Foster City, Calif) with added reverse transcriptase and RNAase inhibitor using ABI Prism 7000 Sequence detector system (Applied Biosystems). The following conditions were used to run the reaction: 30 minutes at $48^{\circ} \mathrm{C}, 10$ minutes at $95^{\circ} \mathrm{C}$ for 1 cycle, and $15 \mathrm{sec}-$ onds at $95^{\circ} \mathrm{C}$ and 1 minute at $60^{\circ} \mathrm{C}$ for 40 cycles. Relative expression levels of the genes were analyzed using $2^{-\mathrm{ddCT}}$ method in comparison to the 
expression of the endogenous control gene GAPDH (Invitrogen). Each sample was analyzed in duplicates.

\section{Multiplex Florescent-Bead Immunoassay}

A multiplex florescent-bead immunoassay was performed to detect cytokine concentrations from the serum samples $(\mathrm{n}=7$ each, patients with $\mathrm{DM}$ and patients without DM) diluted 1:1 in buffer provided with the kit (Invitrogen). Cytokines were analyzed in duplicates. Standard curves were generated for each cytokine by using the reference cytokine concentrations supplied by the manufacturer. Raw data (mean fluorescent intensity) were analyzed by Beadview Software (Upstate Inc, Charlottesville, Va) to obtain concentration values.

\section{Statistical Analysis}

RMA statistical software was used to calculate fold-change for analyzing differential gene expression by microarray. For cytokine comparison analysis, one-way analysis of variance in combination with standard $t$ test (SYSTAT Software Inc., San Jose, Calif) was used to calculate significance.

\section{RESULTS}

\section{Clinical Characteristics and Outcomes}

There were no other significant differences between patients with DM and patients without DM in terms of demographics and perioperative data except for diabetes, hyperglycemia, and hemoglobin A1C $(P<.001$; Table 1$)$. All patients in the study had successful cardiac surgery and recovery (no reoperations or deaths). Clinical outcomes showed significant differences for length of hospital stay post-CPB/cardioplegic arrest $(P=.04)$ and weight gain $(P=.001)$ but not for atrial fibrillation and sternal wound infections between the 2 patient populations (Table 1). No statistically significant difference in postoperative diuretic, blood product, or intravenous fluid administration was detected between the groups. However, the average fluid balance on the day of surgery was $3562 \pm 725.1$ and 3582 $\pm 1042(P=.99)$ and on postoperative day 4 was $+600 \pm$ 1027.8 and $-108 \pm 903.2(P=.6)$, respectively, for patients with DM versus patients without DM. The fluid balance is a crude estimate of fluid homeostasis as the exact measurement of fluid gain and loss through bowel movements, food intake, and insensible losses is difficult, and weight gain more accurately represents the cumulative fluid retention.

\section{VEGF Expression}

VEGF, a multifunctional cytokine known to be involved in inflammatory responses and weight gain, was significantly increased following $\mathrm{CPB}$ when measured at 6 hours post-CPB/ cardioplegic arrest only in patients with DM $(P<.05)$. The expression of VEGF tended to be higher even after 4 days postsurgery when compared with presurgery only in patients with DM, but this difference was not significant. In the case of patients without DM, the variations in VEGF expression at 6 hours or 4 days post-CPB/cardioplegic arrest compared with presurgery were minimal (Figure 1, A). The relative difference in VEGF expression (presurgery vs 6 hours post- $\mathrm{CPB}$ /cardioplegic arrest) was significantly higher in
TABLE 1. Patient characteristics

\begin{tabular}{|c|c|c|c|}
\hline Characteristics & DM & ND & $P$ value \\
\hline Age $(y)^{*}$ & $62.4 \pm 2.1$ & $67.7 \pm 3.2$ & .2 \\
\hline Men/women (n) & $4 / 3$ & $6 / 1$ & 4 \\
\hline $\operatorname{HbA1C}(\mathrm{mg} / \mathrm{dL}) *$ & $12 \pm 1.4$ & $5.6 \pm 0.3$ & $<.001$ \\
\hline Blood glucose levels $(\mathrm{mg} / \mathrm{dL}) *$ & $292.9 \pm 21.9$ & $119 \pm 12.8$ & $<.001$ \\
\hline Obesity (BMI > 30) & $3 / 7(42.8 \%)$ & $4 / 7(57.1 \%)$ & .7 \\
\hline $\begin{array}{l}\text { History of } \\
\text { hypercholesterolemia } \\
\text { with/without treatment (n) }\end{array}$ & $7(100 \%)$ & $5(71 \%)$ & .4 \\
\hline Hypertension on admission (n) & $2(28.5 \%)$ & $1(14.3 \%)$ & .7 \\
\hline $\begin{array}{l}\text { Chronic renal insufficiency } \\
\quad(\text { creatinine }>2), n\end{array}$ & $1(14.3 \%)$ & $1(14.3 \%)$ & NS \\
\hline $\begin{array}{l}\text { Aminocaproic acid } \\
\text { (Amicar, } 100 \mathrm{mg} / \mathrm{kg} \\
\text { load, } 5-\mathrm{g} \text { pump prime, } \\
30 \mathrm{mg} \cdot \mathrm{kg}^{-1} \cdot \mathrm{h}^{-1} \text { infusion) }\end{array}$ & 4 & 4 & NS \\
\hline $\begin{array}{l}\text { Aprotinin } \\
\text { (half-Hammersmith } \\
\text { dose at } 1,000,000 \mathrm{KIU} \text { ) }\end{array}$ & 3 & 3 & NS \\
\hline CABG only (n) & 5 & 5 & NS \\
\hline No. of grafts performed (n) & 3 & 3 & NS \\
\hline $\mathrm{CABG}+$ valve procedures $(\mathrm{n})$ & 2 & 2 & NS \\
\hline Crossclamp time $(\mathrm{min})^{*}$ & $64.3 \pm 17.6$ & $54 \pm 8.7$ & .6 \\
\hline Duration of CPB (min)* & $98.7 \pm 17.3$ & $73.7 \pm 8.9$ & .2 \\
\hline Atrial fibrillation (n) & $2(28.5 \%)$ & $2(28.5 \%)$ & NS \\
\hline Length of stay post surgery (d)* & $10 \pm 1$ & $6 \pm 0.4$ & .04 \\
\hline Weight gain $1 \mathrm{~d}$ post-CPB $(\mathrm{kg})^{*}$ & $6.4 \pm 1.2$ & $6.5 \pm 1.5$ & .8 \\
\hline Weight gain 4 days post-CPB $(\mathrm{kg})^{*}$ & $5 \pm 0.5$ & $2.5 \pm 0.4$ & .001 \\
\hline Intraoperative insulin $(\mathrm{U} / \mathrm{h})^{*}$ & 7 & 0 & .009 \\
\hline Postoperative insulin $(\mathrm{U} / \mathrm{h})^{*}$ & 4 & 3 & .04 \\
\hline
\end{tabular}

$B M I$, Body mass index; $C A B G$, coronary artery bypass grafting; $C P B$, cardiopulmonary bypass; HbAlC, hemoglobin A1C; $N S$, no significance. *Data expressed as mean \pm standard error of the mean.

patients with DM when compared with patients without DM $(P<.02)$. There was a trend toward higher relative rise of VEGF expression even at 4 days post- $\mathrm{CPB} /$ cardioplegic arrest in patients with DM compared with patients without DM, but this trend was not statistically significant (Figure 1,B).

\section{HGF Expression}

HGF, another multifunctional cytokine known to be involved in inflammation and hypervascular permeability, was enhanced significantly 6 hours post- $\mathrm{CPB} /$ cardioplegic arrest in both patients with DM and patients without DM $(P<.05)$. Also, the expression levels were significantly higher $(P<.01)$ in patients with DM as compared with patients without DM 6 hours post-CPB/cardioplegic arrest. The expression of HGF tended to be higher than presurgery levels even at 4 days post-CPB/cardioplegic arrest in both the patient populations but with no statistical significance (Figure 2, A). Similar to VEGF expression, the relative difference in HGF expression (presurgery vs 6 hours post$\mathrm{CPB} /$ cardioplegic arrest) was significantly higher in patients with DM $(P<.02)$ compared with patients without DM (Figure 2, B). The relative rise between patients with DM 

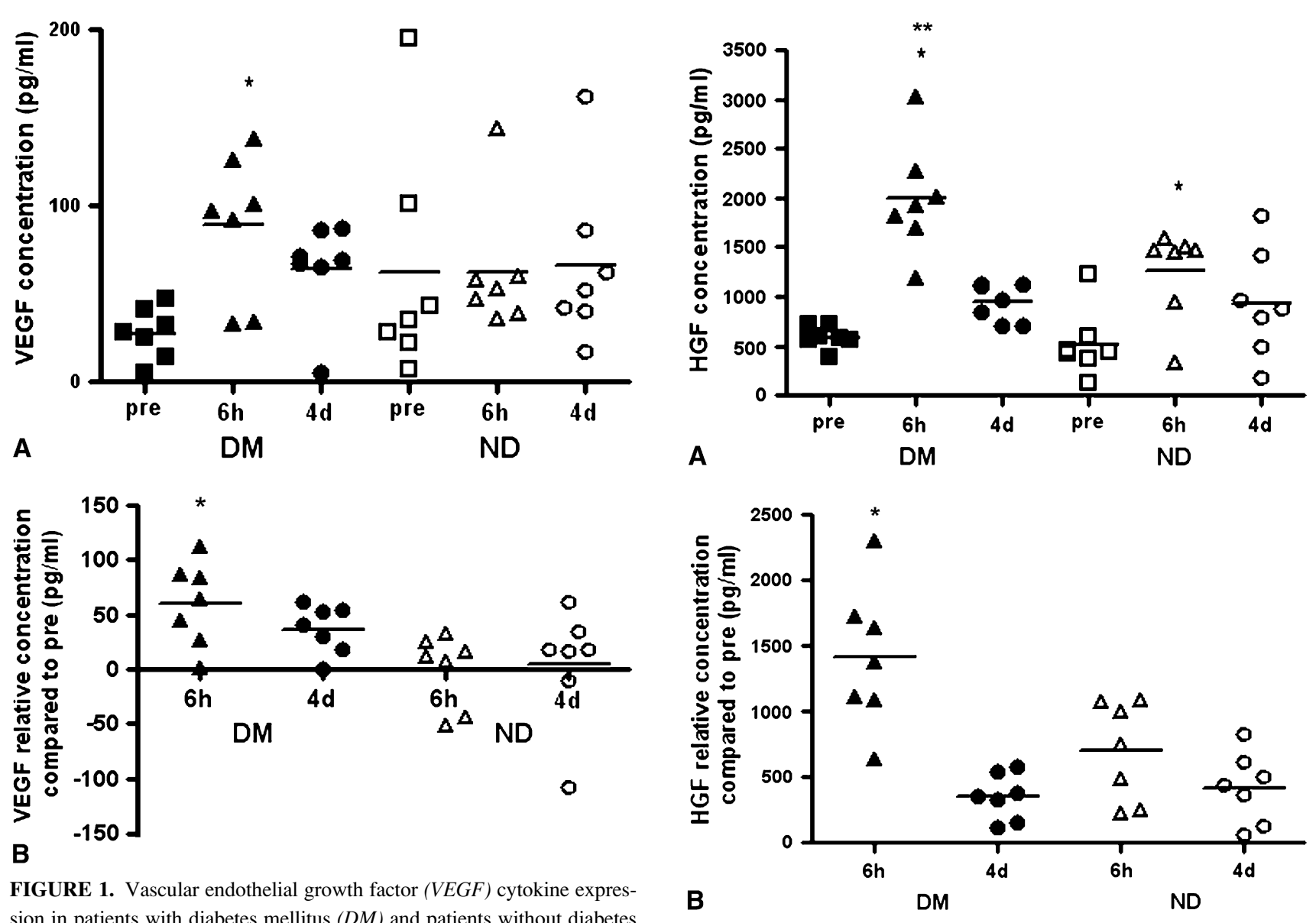

sion in patients with diabetes mellitus $(D M)$ and patients without diabetes mellitus $(N D)$ presurgery (pre), 6 hours postsurgery ( $6 h)$, and 4 days postsurgery $(4 d)$. A, VEGF concentrations $(\mathrm{pg} / \mathrm{mL})$ in the serum of patients with diabetes mellitus and patients without diabetes mellitus $(n=7$ each) at the indicated time points. $* P<.05$ in cytokine expression presurgery compared with 6 hours or 4 days for the specific population (patients with diabetes mellitus or patients without diabetes mellitus). B, Relative VEGF concentration presurgery compared with 6 hours or 4 days postsurgery. $* P<.05$ in relative cytokine expression in patients with diabetes mellitus versus patients without diabetes mellitus at that time point. Closed squares, Presurgery in patients with diabetes mellitus; closed triangles, 6 hours postsurgery in patients with diabetes mellitus; closed circles, 4 days postsurgery in patients with diabetes mellitus; open squares, presurgery in patients without diabetes mellitus; open triangles, 6 hours postsurgery in patients without diabetes mellitus; and open circles, 4 days postsurgery in patients without diabetes mellitus.

and patients without DM was transient and returned to baseline levels when assessed at 4 days post-CPB/cardioplegic arrest (Figure 2, B). No significant differences in expression of other growth factors, such as epidermal growth factor and fibroblast growth factor, were observed between patients with DM and patients without DM at all time points tested presurgery compared with post-CPB (data not shown).

\section{Hypoxia Signaling Pathway}

In correlation with VEGF and HGF expression, HIF- $1 \alpha$, a crucial regulator of hypoxia signaling, and other HIF- $1 \alpha$ -

FIGURE 2. Hepatocyte growth factor $(H G F)$ cytokine expression in patients with diabetes mellitus $(D M)$ and patients without diabetes mellitus (ND) presurgery (pre), 6 hours postsurgery (6h), and 4 days postsurgery (4d). A, HGF concentrations $(\mathrm{pg} / \mathrm{mL})$ in the serum of patients with diabetes mellitus and patients without diabetes mellitus $(n=7$ each) at the indicated time points. ${ }^{*} P<.05$ in cytokine expression presurgery compared with 6 hours or 4 days postsurgery for the specific population (patients with diabetes mellitus or patients without diabetes mellitus). $* * P<.05$ in concentration of HGF in patients with diabetes mellitus versus patients without diabetes mellitus at that time. B, Relative HGF concentration presurgery compared with 6 hours or 4 days postsurgery. $* P<.05$ in the relative cytokine expression in patients with diabetes mellitus versus patients without diabetes mellitus at that time. Closed squares, presurgery in patients with diabetes mellitus; closed triangles, 6 hours postsurgery in patients with diabetes mellitus; closed circles, 4 days postsurgery in patients with diabetes mellitus; open squares, presurgery in patients without diabetes mellitus; open triangles, 6 hours postsurgery patients without diabetes mellitus; and open circles, 4 days postsurgery patients without diabetes mellitus.

related transcription factors such as E1A binding protein p300 (EP300) and cyclic adenosine monophosphate response element binding protein (CREB) were significantly up-regulated greater than twofold only in patients with DM $(P<.001) 4$ days post-CPB/cardioplegic arrest (Figure 3). Validation of these exclusively up-regulated genes in patients with DM involved in hypoxia signaling pathway 


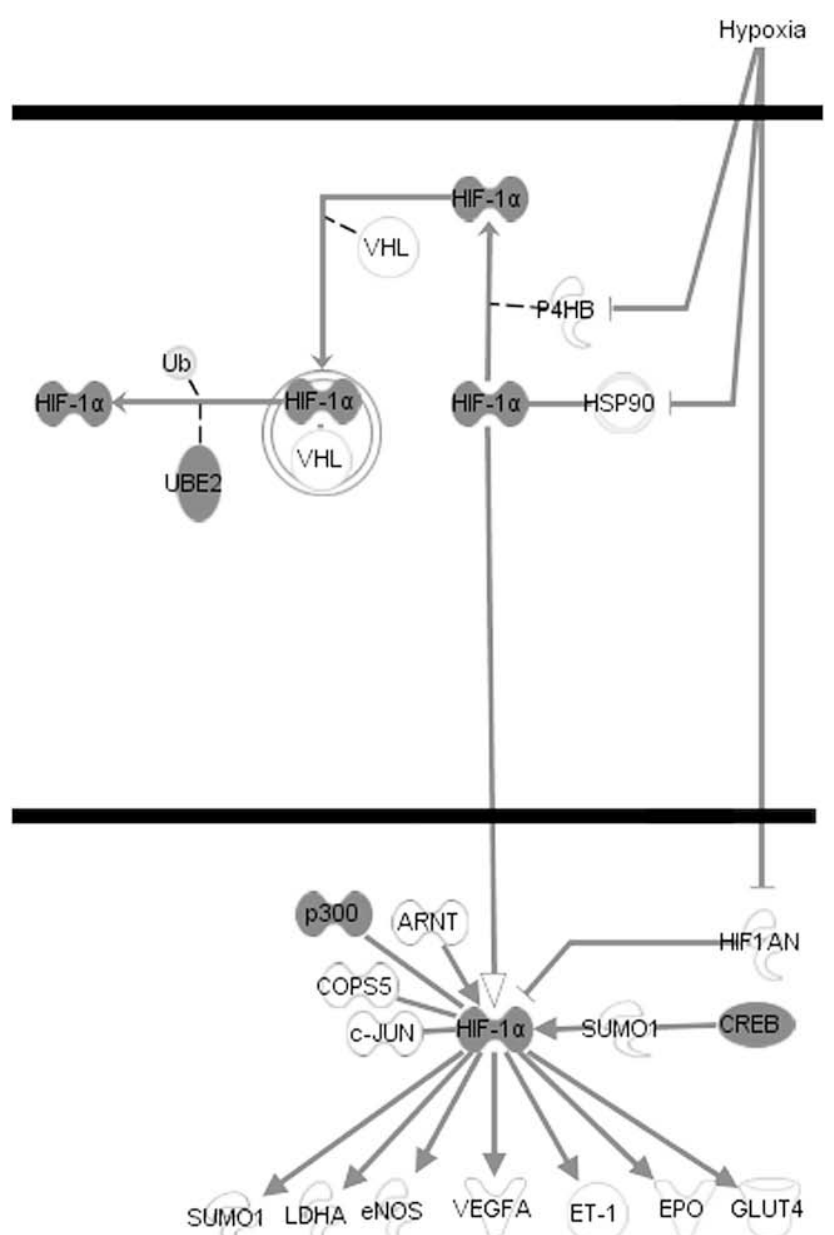

FIGURE 3. Hypoxia signaling pathway in patients with diabetes mellitus 4 days post-cardiopulmonary bypass/cardioplegic arrest. Dark gray indicates up-regulated genes, and light gray represents down-regulated genes in patients with diabetes mellitus. None of the genes in this pathway were up- or down- regulated in patients without diabetes mellitus at 4 days post-cardiopulmonary bypass/cardioplegic arrest. Data were analyzed through the use of Ingenuity Pathway analysis (Ingenuity Systems, www. ingenuity.com).

was confirmed by quantitative real-time PCR analysis (Figure 4). In contrast, the above hypoxia-related genes were not up- or down-regulated in patients without DM. The genes involved in downstream VEGF signaling such as KRAS and SOS were also significantly up-regulated only in patients with DM (data not shown). SHC, an adaptor protein known to mediate HIF- $1 \alpha$ induction under hypoxic conditions, was down-regulated only in patients without DM (data not shown). Insulin-like growth factor 1 receptor (IGF1R) was significantly expressed exclusively in patients with DM $(P<.001) 4$ days post-CPB (data not shown).

\section{Correlation between Serum VEGF Levels and CRP}

CRP, a marker of acute inflammation and a contributor to inflammatory diseases including arteriosclerosis as well as

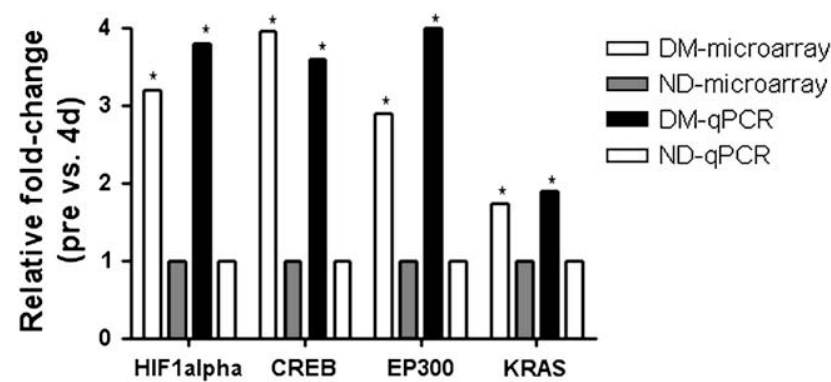

FIGURE 4. Validation and comparison of up-regulated genes (exclusively in patients with diabetes mellitus) in the hypoxia-signaling pathway using quantitative real-time polymerase chain reaction versus microarray analysis. $* P<.001$ between patients with diabetes mellitus versus patients without diabetes mellitus. $D M$, Patients with diabetes mellitus; $N D$, patients without diabetes mellitus.

other cardiovascular diseases, was elevated to different levels post-CPB in patients with DM and patients without DM. CRP expression was significantly increased following $\mathrm{CPB}$ when measured at 6 hours post-CPB/cardioplegic arrest only in patients with $\mathrm{DM}(P<.05$; Figure $5, A)$. The relative expression of CRP was significantly higher in patients with DM when compared with patients without DM at 6 hours post-CPB/cardioplegic arrest (Figure 5, B). CRP serum levels persistently increased post-CPB/cardioplegic arrest and were significantly enhanced at 4 days in both patients with DM and patients without DM (Figure 5). No positive correlation was observed between VEGF and $\mathrm{CRP}$ at any tested time in both the patient populations.

\section{DISCUSSION}

In this study, the serum VEGF levels increased significantly in patients with DM compared with patients without $\mathrm{DM}$ at 6 hours post- $\mathrm{CPB} /$ cardioplegic arrest. Furthermore, the relative increase in VEGF and HGF levels presurgery versus 6 hours post-CPB/cardioplegic arrest was significantly higher in patients with DM when compared with patients without DM. The results of the current study are primarily a reflection of patients with poorly controlled diabetes, and further investigation is required to extend the results of this study to patients with better preoperative glucose control.

Although the levels of VEGF were not significantly different between presurgery and 4 days post-cardiac surgery, a trend toward higher VEGF levels was observed at least until 4 days post-CPB/cardioplegic arrest in patients with DM. Several studies reported up-regulation of VEGF and HGF expression under hypoxic conditions in many disease settings including diabetic retinopathy. ${ }^{7}$ HGF is also known to function as a potent inducer of VEGF expression by human epidermal keratinocytes ${ }^{23}$ and its receptor flk 1 in endothelial cells. ${ }^{24}$ VEGF and HGF growth factors were extensively studied in relation to diabetic retinopathy and are reported as major contributors to retinal edema. 

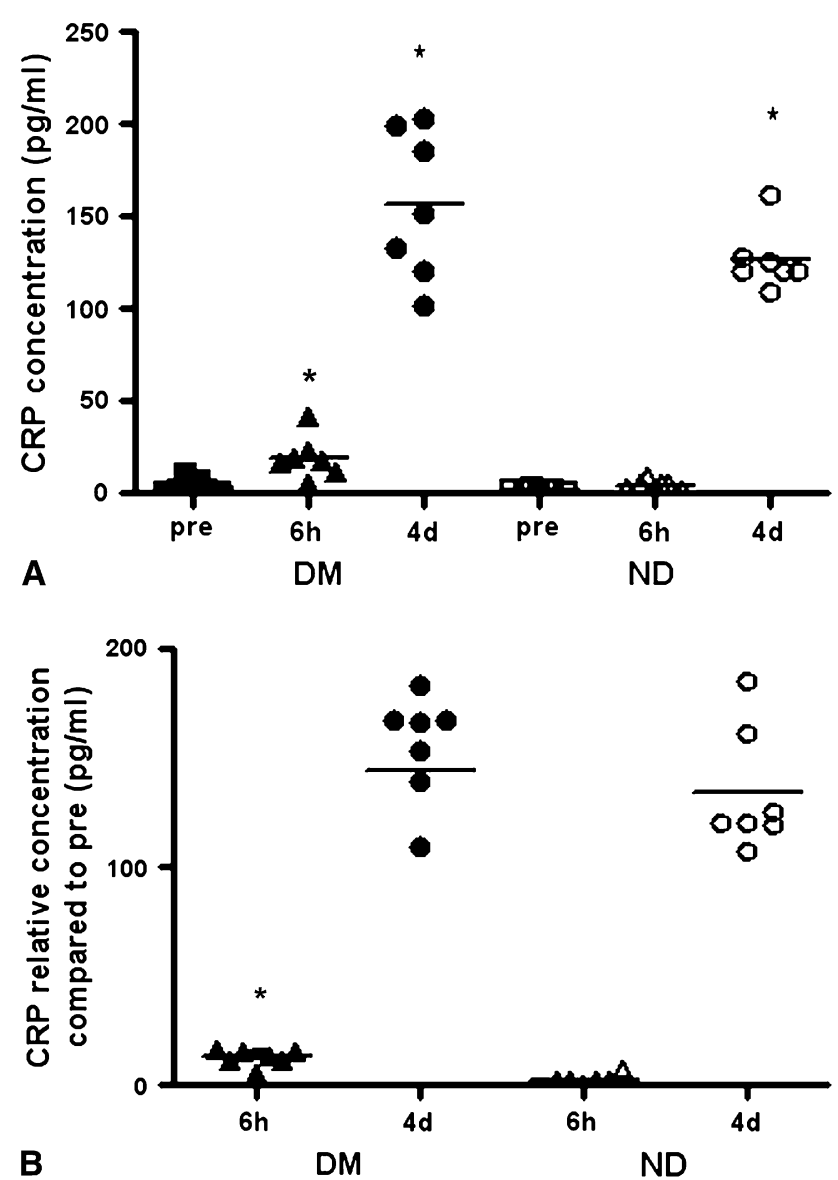

FIGURE 5. C-reactive protein $(C R P)$ cytokine expression profile in patients with diabetes mellitus $(D M)$ and patients without diabetes mellitus $(N D)$ presurgery (pre), 6 hours postsurgery (6h), and 4 days postsurgery $(4 d)$. A, CRP concentrations $(\mathrm{pg} / \mathrm{mL})$ in the serum of patients with diabetes mellitus and patients without diabetes mellitus $(\mathrm{n}=7$ each) at the indicated time points. $* P<.05$ in cytokine expression presurgery compared with 6 hours or 4 days postsurgery for the specific population (patients with diabetes mellitus or patients without diabetes mellitus). B, Relative CRP concentration presurgery compared with 6 hours or 4 days postsurgery. $* P<.05$ in relative cytokine expression in patients with diabetes mellitus versus patients without diabetes mellitus at that time point. Closed squares, Presurgery in patients with diabetes mellitus; closed triangles, 6 hours postsurgery in patients with diabetes mellitus; closed circles, 4 days postsurgery in patients with diabetes mellitus; open squares, presurgery in patients without diabetes mellitus; open triangles, 6 hours postsurgery in patients without diabetes mellitus; and open circles, 4 days postsurgery in patients without diabetes mellitus.

VEGF is a multifunctional cytokine known to be involved in endothelial cell proliferation, ${ }^{16}$ chemotaxis of macrophages and vascular smooth muscle cells,${ }^{17}$ and induction of adhesion molecules (vascular cell adhesion molecule and intercellular adhesion molecule $)^{18}$ and metalloproteinase synthesis. ${ }^{19}$ Acute systemic hypoxia can induce VEGF expression, which regulates leukocyte extravasation into tissues followed by fluid accumulation and thus induces overall weight gain in patients after cardiac surgery. In this study, clinical outcomes such as length of hospitalization (10 vs 6 days postsurgery; $P=.04$ ) and weight gain (5 vs $2.5 \mathrm{~kg} 4$ days postsurgery; $P=.001$ ) were significantly greater for patients with DM compared with patients without DM, which may be a consequence of higher and sustained VEGF serum levels in the DM population. Preoperative glucose control and/or restoration to a normal hemoglobin A1C may ameliorate the effects of $\mathrm{CPB}$ upon postoperative recovery. In conjunction with VEGF protein expression levels, CRP, a well-studied marker for systemic inflammation, was also expressed at higher levels in patients with DM compared with patients without DM. CRP could serve as another potential marker for weight gain in patients with DM. Consideration must be given to strategies that reduce the inflammatory response in diabetic patients having cardiac surgery. Such strategies include pharmacologic manipulation and performance of off-pump coronary bypass in appropriate patients.

In correlation with VEGF cytokine levels, HIF- $1 \alpha$, a crucial regulator of hypoxia signaling pathway and thus a potent stimulator of VEGF, was significantly increased only in patients with DM 4 days post-CPB/cardioplegic arrest. CREB and EP300, which act as coactivators of HIF- $1 \alpha$, were also significantly expressed only in patients with DM 4 days post-CPB/cardioplegic arrest. UBE2, a ubiquitin protein known to catalyze hydroxylated human HIF- $1 \alpha$ protein and other ubiquitin proteins (Ub), was up-regulated only in patients with DM. ${ }^{25}$ Some of the crucial genes that are involved in the downstream signaling of VEGF such as KRAS and its activator gene, SOS, were also up-regulated only in patients with DM 4 days post-CPB/cardioplegic arrest. VEGF signaling induces several genes including VEGF, which acts in an autocrine manner. ${ }^{26}$ In contrast, the genes regulated in patients without DM (such as SHC and PKHR) were down-regulated, thus negatively impacting the VEGF signaling pathway. Taken together, patients with DM had an overall increase in regulation of the HIF$1 \alpha$ pathway at multiple levels.

In addition, IGF1R, which is known to induce VEGF expression by positively regulating HIF- $1 \alpha$ expression, was also significantly expressed only in patients with DM 4 days post-CPB/cardioplegic arrest. ${ }^{27}$ Therefore, the differential expression of growth factors and their signaling related genes between patients with DM versus patients without DM could contribute to the difference in observed clinical outcomes in the 2 patient populations.

There are some limitations to our study. First, the study was performed with few patient samples from each group (patients with DM and patients without DM, $\mathrm{n}=7$ each). Based upon an effect size of 1.2 and power of 0.8 , we estimated a sample size of 12 in each group. However, given the large differences noted between the diabetic versus nondiabetic groups, the differences between the 2 groups were 
significant at a sample size of 7 in each group. This study is underpowered to detect an effect size less than 1.5. Second, more time points need to be analyzed to get a better understanding of growth factor functions post-cardiac surgery in patients with DM and patients without DM. Previous studies suggest a peak regulation of several inflammatory and apoptotic genes within 6 hours post-CPB. Therefore, 6 hours postsurgery was considered as a time point of interest. Because the inflammatory response is expected to abate by 4 days postsurgery, gene regulation was assessed at 4 days post-CPB. Another limitation of the study is that although there was no significant difference in the duration of $\mathrm{CPB}$ between patients with DM and patients without DM, there was a trend toward longer bypass time in the patients with DM.

Although our study implies the possibility that VEGF may have some role in developing edema and fluid retention in patients with DM, it could be difficult to predict if increases in plasma VEGF adversely influence weight gain in patients with DM or could be useful for therapeutic angiogenesis for ischemic artery disease, which is often seen in patients with type 2 diabetes. ${ }^{28}$ However, the systemic edema and weight gain may partially be attributed to VEGF function along with the expression of other inflammatory markers such as CRP. Further studies may be required to clarify these speculations. In conclusion, we hypothesize that the differential expression of VEGF and HGF and up-regulation of the hypoxia-regulated master gene HIF- $1 \alpha$ in patients with DM versus patients without DM may reflect more severe postsurgical complications in patients with DM.

We acknowledge the genomics core facility at the Beth Israel Deaconess Medical Center (www.bidmcgenomics.org) for their assistance and expertise in the gene array analysis. Dr Manoj Bhasin has performed the statistical analysis for the microarray data.

\section{References}

1. Lauruschkat AH, Arnrich B, Albert AA, Walter JA, Amann B, Rosendahl UP, et al. Prevalence and risks of undiagnosed diabetes mellitus in patients undergoing coronary artery bypass grafting. Circulation. 2005;112:2397-402.

2. Furnary AP, Zerr KJ, Grunkemeier GL, Starr A. Continuous intravenous insulin infusion reduces the incidence of deep sternal wound infection in diabetic patients after cardiac surgical procedures. Ann Thorac Surg. 1999;67:352-60; discussion 60-2.

3. Krinsley JS. Association between hyperglycemia and increased hospital mortality in a heterogeneous population of critically ill patients. Mayo Clin Proc. 2003;78: 1471-8.

4. Eberhardt F, Mehlhorn U, Larose K, De Vivie ER, Dhein S. Structural myocardial changes after coronary artery surgery. Eur J Clin Invest. 2000;30:938-46.

5. Semenza GL, Agani F, Feldser D, Iyer N, Kotch L, Laughner E, et al. Hypoxia, HIF-1, and the pathophysiology of common human diseases. Adv Exp Med Biol. 2000;475:123-30

6. Poulaki V, Mitsiades CS, McMullan C, Sykoutri D, Fanourakis G, Kotoula V, et al. Regulation of vascular endothelial growth factor expression by insulinlike growth factor I in thyroid carcinomas. J Clin Endocrinol Metab. 2003;88: 5392-8.

7. Clermont AC, Cahill M, Salti H, Rook SL, Rask-Madsen C, Goddard L, et al. Hepatocyte growth factor induces retinal vascular permeability via MAP-kinase and PI-3 kinase without altering retinal hemodynamics. Invest Ophthalmol Vis Sci. 2006;47:2701-8.

8. Gillis P, Savla U, Volpert OV, Jimenez B, Waters CM, Panos RJ, et al. Keratinocyte growth factor induces angiogenesis and protects endothelial barrier function. J Cell Sci. 1999;112(Pt 12):2049-57.

9. Tanabe K, Tokuda H, Takai S, Matsushima-Nishiwaki R, Hanai Y, Hirade K, et al. Modulation by the steroid/thyroid hormone superfamily of TGF-beta-stimulated VEGF release from vascular smooth muscle cells. J Cell Biochem. 2006; 99:187-95.

10. Yoshino Y, Aoyagi M, Tamaki M, Duan L, Morimoto T, Ohno K. Activation of p38 MAPK and/or JNK contributes to increased levels of VEGF secretion in human malignant glioma cells. Int J Oncol. 2006;29:981-7.

11. Santos SC, Miguel C, Domingues I, Calado A, Zhu Z, Wu Y, et al. VEGF and VEGFR-2 (KDR) internalization is required for endothelial recovery during wound healing. Exp Cell Res. 2007;313:1561-74.

12. Sellke FW, Ruel M. Vascular growth factors and angiogenesis in cardiac surgery. Ann Thorac Surg. 2003;75:S685-90.

13. Kirk SL, Karlik SJ. VEGF and vascular changes in chronic neuroinflammation. J Autoimmun. 2003;21:353-63.

14. Lu K, Zhou Y, Kaufman K, Mott R, Ma JX. Rat strain-dependent susceptibility to ischemia-induced retinopathy associated with retinal vascular endothelial growth factor regulation. J Mol Endocrinol. 2007;38:423-32.

15. Said HM, Hagemann C, Staab A, Stojic J, Kuhnel S, Vince GH, et al. Expression patterns of the hypoxia-related genes osteopontin, CA9, erythropoietin, VEGF and HIF-1alpha in human glioma in vitro and in vivo. Radiother Oncol. 2007; 83:398-405.

16. Hiroishi G, Murata T, Ishibashi T. Effect of thiazolidinedione on the proliferation of bovine retinal endothelial cells stimulated by vascular endothelial cell growth factor. Jpn J Ophthalmol. 2007;51:21-6.

17. Tsuchihashi S, Ke B, Kaldas F, Flynn E, Busuttil RW, Briscoe DM, et al. Vascula endothelial growth factor antagonist modulates leukocyte trafficking and protects mouse livers against ischemia/reperfusion injury. Am J Pathol. 2006;168:695-705.

18. Kim I, Moon SO, Kim SH, Kim HJ, Koh YS, Koh GY. Vascular endothelial growth factor expression of intercellular adhesion molecule 1 (ICAM-1), vascular cell adhesion molecule 1 (VCAM-1), and E-selectin through nuclear factor-kappa B activation in endothelial cells. $J$ Biol Chem. 2001;276:7614-20.

19. Noda K, Ishida S, Shinoda H, Koto T, Aoki T, Tsubota K, et al. Hypoxia induces the expression of membrane-type 1 matrix metalloproteinase in retinal glial cells. Invest Ophthalmol Vis Sci. 2005;46:3817-24.

20. Verma S, Badiwala MV, Weisel RD, Li SH, Wang CH, Fedak PW, et al. C-reactive protein activates the nuclear factor-kappaB signal transduction pathway in saphenous vein endothelial cells: implications for atherosclerosis and restenosis. J Thorac Cardiovasc Surg. 2003;126:1886-91.

21. Yang H, Nan B, Yan S, Li M, Yao Q, Chen C. C-reactive protein decreases expression of VEGF receptors and neuropilins and inhibits VEGF165-induced cell proliferation in human endothelial cells. Biochem Biophys Res Commun. 2005;333:1003-10.

22. Voisine P, Ruel M, Khan TA, Bianchi C, Xu SH, Kohane I, et al. Differences in gene expression profiles of diabetic and nondiabetic patients undergoing cardiopulmonary bypass and cardioplegic arrest. Circulation. 2004;110(11 Suppl. 1):II280-6.

23. Gille J, Khalik M, Konig V, Kaufmann R. Hepatocyte growth factor/scatter factor (HGF/SF) induces vascular permeability factor (VPF/VEGF) expression by cultured keratinocytes. J Invest Dermatol. 1998;111:1160-5.

24. Wojta J, Kaun C, Breuss JM, Koshelnick Y, Beckmann R, Hattey E, et al. Hepatocyte growth factor increases expression of vascular endothelial growth factor and plasminogen activator inhibitor-1 in human keratinocytes and the vascular endothelial growth factor receptor flk-1 in human endothelial cells. Lab Invest. 1999; 79:427-38.

25. Kamura T, Sato S, Iwai K, Czyzyk-Krzeska M, Conaway RC, Conaway JW. Activation of HIF1alpha ubiquitination by a reconstituted von Hippel-Lindau (VHL) tumor suppressor complex. Proc Natl Acad Sci U S A. 2000;97:10430-5.

26. Pal S, Datta K, Khosravi-Far R, Mukhopadhyay D. Role of protein kinase Czeta in Ras-mediated transcriptional activation of vascular permeability factor/vascular endothelial growth factor expression. J Biol Chem. 2001;276:2395-403.

27. Poulaki V, Joussen AM, Mitsiades N, Mitsiades CS, Iliaki EF, Adamis AP. Insulin-like growth factor-I plays a pathogenetic role in diabetic retinopathy. Am J Pathol. 2004;165:457-69.

28. Yamakawa K, Hosoi M, Koyama H, Tanaka S, Fukumoto S, Morii H, et al. Peroxisome proliferator-activated receptor-gamma agonists increase vascular endothelial growth factor expression in human vascular smooth muscle cells. Biochem Biophys Res Commun. 2000;271:571-4. 